\title{
TEST OF THREE POINT OF CARE DEVICES FOR NEONATAL GLYCAEMIA MEASUREMENT
}

N. Piol, C. Stadelmann Diaw, J. Urfer, D. Werner, M. Roth-Kleiner

Centre Hospitalier Universitaire Vaudois, Lausanne, Switzerland

Background: Neonatal hypoglycaemia is a major cause of acute morbidity and may induce neurodevelopmental impairment. Clinical symptoms are unspecific or even absent. Therefore, diagnosis depends on precise and accurate methods for glycaemia measurement. Point-of-care testing (POCT) handheld devices, widely used for self-testing in diabetic patients, have shown efficiency in adults. But as newborns have physiologically low glycaemia, precision and accuracy of such glucometers need to be validated for neonatal use.

Method: In this prospective study, we tested three POCT devices (A: Precision Xceed ${ }^{\mathrm{TM}}$, Abbott; B: Elite ${ }^{\mathrm{TM}}$ XL, Bayer; C: Aviva Nano ${ }^{\mathrm{TM}}$, Roche) against the hexokinase reference method (Modular P system, Roche). Agreement between methods was analyzed using Passing\&Bablok fits and bias plots by modified BlandAltman method. Accuracy was checked by ISO 15197 criteria.

Results: A total of 774 paired glycaemia values (range $0.3-13 \mathrm{mmol} / \mathrm{l}$ ) were obtained over a one-year study period by one of the three devices and the reference method. Passing\&Bablok fits were $\mathbf{A}=1.11$ xReference $0.27 \mathrm{mmol} / 1 ; \mathbf{B}=1.0 x$ Reference $-0.10 \mathrm{mmol} / 1$ and $\mathbf{C}=1.00 x$ Reference $+0.0 \mathrm{mmol} / 1$. Mean bias were +0.29 , 0.02 and $+0.04 \mathrm{mmol} / 1$ for $\mathbf{A}, \mathbf{B}$ and $\mathbf{C}$ respectively. Interestingly, ISO 15197 accuracy criteria were fulfilled only by $\mathbf{A}$, and just for values $<4.2 \mathrm{mmol} / 1$.

Conclusions: Due to their easy use, their rapid result availability and small blood volume needs, POCT devices are indispensable for neonatal glycaemia measurements. However, it is crucial to know their specificities and limitations for interpretation of results. With regard to neonatal hypoglycaemia screening, A might be slightly more advantageous than $\mathbf{B}$ and $\mathbf{C}$, as it presents lowest risk to miss hypoglycaemia. 